\title{
Accuracy of clinical criteria and an immunochromatographic strip test for dengue diagnosis in a DENV-4 epidemic
}

\author{
Sibelle Nogueira Buonora ${ }^{*}$, Sonia Regina Lambert Passos ${ }^{1}$, Cleber Nascimento do Carmo², \\ Fernanda Moisés Quintela ${ }^{1}$, Diana Neves Rodrigues de Oliveira', Flavia Barreto dos Santos ${ }^{4}$, \\ Yara Hahr Marques Hökerberg ${ }^{1}$, Rita Maria Ribeiro Nogueira ${ }^{4}$ and Regina Paiva Daumas ${ }^{3}$
}

\begin{abstract}
Background: Early diagnosis of dengue infection is important for decision-making and timely implementation of therapeutic measures. Although rapid NS1 assays have been used for dengue diagnosis since 2008, their performance in DENV-4 cases has not yet been fully assessed.

Methods: We evaluated the accuracy of NS1 Bioeasy ${ }^{\text {TM }}$ immunochromatographic strip test and of three clinical criteria for dengue diagnosis. Patients presenting at an emergency care center within $72 \mathrm{~h}$ of an acute febrile illness during the 2013 DENV-4 epidemic in Rio de Janeiro were consecutively enrolled for clinical and laboratory evaluation. We classified patients as suspected dengue or not according to three clinical criteria: WHO 2009, WHO 1997, and INI-FIOCRUZ. Dengue diagnosis was defined by RNA detection using RT-PCR and the negative cases were negative for all dengue serotypes and also Platelia ${ }^{\mathrm{TM}}$ NS1 ELISA. We obtained accuracy indices for NS1 Bioeasy ${ }^{\mathrm{TM}}$ alone and in combination with the clinical criteria.

Results: RT-PCR for DENV-4 was positive in 148 out of 325 patients. Positive likelihood ratio, sensitivity, and specificity of NS1 Bioeasy ${ }^{\text {TM }}$ with WHO 2009, WHO 1997, and INI-FIOCRUZ criteria were 22.6 (95 \% Cl 7.2-70.6), $40.6 \%$ (95 \% Cl 32.3-49.3), and 98.2\% (95\% Cl 94.9-99.6); 18.3 (95\% Cl 6.8-49.2), 44.2 (95\% Cl 35.8-52.9), 97.6 (95\% Cl 94.0-99.3); 26.2 (95 \% Cl 6.5-106.5), 29.7 (95 \% Cl 22.4-37.8), 98.9 (95 \% Cl 96.0-99.9), respectively. WHO 1997 clinical criteria presented high sensitivity to rule out disease, but extremely low specificity. INI-FIOCRUZ had moderate sensitivity and specificity, and could target a group to a more specific test.
\end{abstract}

Conclusions: Although the large rates of false negative results using NS1 Bioeasy ${ }^{\mathrm{TM}}$ rapid test advise against its use for triaging (rule out) purposes in DENV-4 epidemics, it could be used as a confirmatory tool in a bedside algorithm.

Keywords: Dengue, Accuracy, Sensitivity, Specificity, Diagnosis, Point-of-care systems, NS1

\section{Background}

Dengue is an acute viral febrile disease mainly transmitted by the Aedes aegypti mosquito. Dengue virus has four serotypes (DENV 1-4) defined by phylogenetic and antigenic characteristics. The immunity resulting from infection is serotype-specific and does not protect the individual against other serotypes [1].

\footnotetext{
* Correspondence: sibellebuonora@gmail.com

'Laboratory of Clinical Epidemiology, Evandro Chagas National Institute of Infectious Diseases, Oswaldo Cruz Foundation, Rio de Janeiro, Brazil Full list of author information is available at the end of the article
}

A recent study estimated that about 390 million cases of dengue occurred in 2010, which is more than three times the World Health Organization (WHO) estimates for that same period [2]. However, only 96 million were symptomatic. Brazil has the highest dengue reporting rates worldwide and between 2000 and 2007, over three million cases were reported in the country, corresponding to approximately $60 \%$ of cases in the Americas [3].

In Brazil, dengue outbreaks have occurred yearly since 1986 and during epidemic seasons the entire health care system is overwhelmed by a three to four-fold increase in patient visits. In the state of Rio de Janeiro, epidemics 
occurred in 1986 [4], 1990 [5], 2000 [6], and 2008 [7] by DENV-1, DENV-2, DENV-3, and by the reemergence of DENV-2, respectively, usually followed by dissemination to other regions of the country. DENV-4 was first identified in this Brazilian state in 2011 [8] and accounted for 218,000 reported cases in the 2013 epidemic.

Early diagnosis is critical because some patients may progress from a mild to a severe disease in a short period of time $[9,10]$. Repeated monitoring of platelet count and hematocrit is recommended, as an abrupt decrease in platelet count is a warning sign and a significant hematocrit increase is an indirect sign of plasma leakage [7]. In 1997, WHO proposed as a dengue clinical case a patient that presented with fever and two or more of the following: headache, retro-orbital pain, myalgia, arthralgia, rash, hemorrhagic manifestations, and leukopenia [11]. However, due to the low specificity (36\%) of these criteria [12], a new set was proposed in 2009 that grouped myalgia and arthralgia in body aches, included nausea and vomiting, and added some warning signs and symptoms [1]. The $80 \%$ sensitivity and $57 \%$ specificity of the newly proposed WHO criteria [12], supports the importance of laboratory confirmation.

A previous study carried out at the Evandro Chagas National Institute of Infectious Diseases, Oswaldo Cruz Foundation (INI-FIOCRUZ) in Rio de Janeiro analyzed clinical and hematological data in ambulatory febrile patients and derived another diagnostic set of clinical criteria for dengue diagnosis [13]. In this study population the prediction rule, based on the presence of conjunctival hyperemia and leukocyte count, shown $81 \%$ sensitivity and $71 \%$ specificity [13].

Dengue laboratory diagnosis can be performed directly, by identifying the virus or its components, or indirectly, through serological tests detecting antibodies produced against the virus. The sensitivity of each method relies on disease duration at the moment of the clinical specimen collection [14]. The indirect methods are the more commonly used but have limited usefulness in the acute dengue diagnosis. IgM peaks occur around the third to fourth day of disease onset, and therefore a second clinical specimen collection is needed around day 14 to confirm the IgM rise and conclusively diagnose the disease [15]. However, in scenarios where the prevalence of secondary dengue is high, such as in Rio de Janeiro State, the duration and magnitude of the IgM response is reduced possibly impairing the accuracy of this serologic parameter [16]. Furthermore, pairing IgM are usually not point-of-care techniques, limiting their usefulness in epidemic scenarios.

During the febrile phase, detection of viral RNA or nonstructural protein-1 (NS1) are the main methods for the disease diagnosis. However, detection of viral RNA by reverse transcriptase-polymerase chain reaction (RT-PCR) is relatively complex and expensive, and is therefore not feasible in a number of health care settings, particularly in epidemic situations. For this reason, the identification of NS1 has been suggested as an alternative because it is present in the virus membrane, is highly stable and is secreted in the human serum during the early phase of dengue infection [15]. The sensitivity of the NS1 ELISA is reported to be greater than $90 \%$ in primary infections and varies from 60 to $80 \%$ in secondary infections $[17,18]$.

The rapid detection of dengue NS1 by immunochromatographic methods represents a potential breakthrough for laboratory case confirmation at early stages of disease in settings with limited infrastructure because they require minimal laboratory expertise and provide results within 15 to $30 \mathrm{~min}$ of specimen reception [4, 15, 19]. This laboratory technique, first described in 2000 and widely used since 2008, has now been tested in all dengue scenarios, especially in DENV-1 and DENV-3 scenarios $[12,15]$. However, DENV-4 samples are still underrepresented [15, 18, 20, 21].

Ferraz, et al. [22], when evaluating mainly Brazilian DENV-1 samples by three different immunochromatographic assays, identified the NS1 Bioeasy ${ }^{\mathrm{mm}}$ as the one with higher sensitivity (63\%) despite the high specificity presented by all three (100\%). Another Brazilian study evaluating accuracy parameters of 4 immunochromatographic tests in a panel of acute DENV-1 to DENV-3 concluded that NS1 Bioeasy $^{\mathrm{Tm}}$ presented an overall $68 \%$ specificity and a $90 \%$ sensitivity which could reach $95 \%$ in a DENV-1 setting [23].

A simple and accurate test to diagnose acute dengue cases in outpatient healthcare settings is still required. Considering this issue, we evaluated the diagnostic accuracy of three clinical dengue diagnostic criteria (WHO 1997, WHO 2009, INI-FIOCRUZ) and NS1 Bioeasy ${ }^{\text {ma }}$ immunochromatographic test in an urgent care center during a DENV-4 epidemic in the city of Rio de Janeiro, Brazil.

\section{Methods \\ Ethical statement}

This prospective cross-sectional diagnostic accuracy study is reported according to the Standards for Reporting of Diagnostic Accuracy Study (STARD) Guideline [24] and was approved by the Research Ethics Committee of the Evandro Chagas National Institute of Infectious DiseasesFIOCRUZ, CAAE 0066.0.009.000-11, on March 23, 2012.

\section{Enrollment, data, and specimen collection}

We conducted the study in the city of Rio de Janeiro, Rio de Janeiro, Brazil, from March to April 2013, during an ongoing dengue epidemic. Adult patients (age $>18$ years) attending a public urgent care center (Unidade de Pronto Atendimento-UPA $24 \mathrm{H}$ ) within $72 \mathrm{~h}$ of onset of an acute 
febrile illness without an evident focus of infection were eligible for the study.

A trained nurse obtained informed consent and prospectively enrolled the patients during business hours 5 days a week. An infectious disease physician collected data on demographic characteristics, symptoms and physical signs using a summarized version of a previously published and tested semi-structured questionnaire [25]. The tourniquet test was not performed and only spontaneous hemorrhagic manifestations were recorded.

We evaluated all patients according to WHO 1997, WHO 2009, and INI-FIOCRUZ clinical criteria defined elsewhere $[1,11,13,26]$.

Blood samples were collected for complete blood count and specific dengue tests including NS1 Bioeasy ${ }^{\mathrm{Tm}}$, RTPCR, Panbio ${ }^{\circ}$ dengue IgM capture ELISA and dengue IgG ELISA, and Platelia $^{\mathrm{ma}}$ Dengue NS1 Ag-ELISA. Acute dengue-4 cases were confirmed by RT-PCR. Serum samples were sent to the Flavivirus Laboratory, a regional reference laboratory for dengue and yellow fever at the Oswaldo Cruz Foundation (FIOCRUZ) where they were frozen at $-70{ }^{\circ} \mathrm{C}$ until using for dengue specific laboratorial tests.

\section{Index tests}

\section{NS1 strip test}

All febrile cases were tested by the NS1 Bioeasy ${ }^{\text {mi }}$ immunochromatographic strip test (Bioeasy ${ }^{\mathrm{mm}}$, Standard Diagnostics INC, Korea). This rapid test was chosen because it has already been used in the state of Rio de Janeiro and in other Brazilian states during dengue outbreaks. However, its performance had not been previously assessed. According to the manufacturer's instruction, the test can be stored at room temperature and can adequately detect the NS1 within 15 min using three drops of whole blood, plasma or serum.

In the immunochromatographic strip test, the NS1 antigen present in the sample will complex with the gold colloidal particles coated with anti-NS1 antibodies. After migration, the complexes will be captured by anti-NS1 antibodies at the test line where a line will appear. The presence of two lines in the cassette's window means a positive result, whereas the absence of the second line means a negative result with an adequate control line, which ensures the test is working properly.

\section{Clinical criteria}

Patients were classified as suspected dengue cases according to three sets of clinical criteria described as follows:

WHO 1997: fever with two or more of the following: headache, retro-orbital pain, myalgia, arthralgia, rash, hemorrhagic manifestations, and leukopenia [11]; WHO 2009: fever with two or more of the following: nausea/ vomiting, rash, pain, leukopenia, and any of the following warning signs: abdominal pain, persistent vomiting, edema, mucosal bleeding, lethargy, hepatomegaly or hemoconcentration associated with a sudden drop in platelet count [1]; and INI-FIOCRUZ: presence of conjunctival redness with leukocytes less than $7500 / \mathrm{mm}^{3}$ or less than 3760 leukocytes $/ \mathrm{mm}^{3}$ independently of other signs or symptoms [13].

Hemoconcentration in a single sample was defined by a hematocrit $>53 \%$ in males or $48 \%$ in females [27]. Leukopenia was defined as a total leucocyte count $\leq 4500$ cells $/ \mathrm{mm}^{3}$ [27].

\section{Reference tests \\ Dengue viral RNA}

In order to identify dengue viral RNA and specifically dengue 4 serotype, we performed RT-PCR according to the protocol described by Lanciotti et al. [28]. This highly specific protocol suggested by WHO [1] was considered the "gold standard" method for dengue- 4 case confirmation in this study. Briefly, consensus primers (D1-5'-TCAA TATGCTGAAACGCGGAGAAACCG-3') and D2 (5'TTGCACCAACAGTCAATGTCTTCAGGTTC-3') were annealed to any of the four dengue serotypes to amplify a 511-bp product in a reverse transcriptase-polymerase reaction. After a second round of amplification (nested PCR) with type-specific primers (TS1 [5' - CGTCTCAGT GATCCGGGGG- 3'], TS2 [5'-CGCCACAAGGGCCAT GAACAG-3'], TS3 [5'-TAACATCATCATGAGACAGA GC-3'] and TS4 [5'-CTCTGTTGTCTTAAACAAGAG A-3']), DNA products specific for each dengue virus serotype were generated.

\section{NS1 antigen detection by ELISA}

The Platelia ${ }^{\mathrm{Tm}}$ Dengue NS1 Ag-ELISA (BioRad Laboratories, France) was used for NS1 antigen capture according to the instructions by the manufacturer.

\section{Immunologic markers}

We performed the Panbio ${ }^{\circ}$ dengue IgM Capture ELISA (Alere $^{\mathrm{mu}}$, Minas Gerais, Brasil) and Dengue Virus IgG DxSelect $^{\text {tux }}$ ELISA (Focus Diagnostics, California, USA) according to the manufacturers' instructions for the qualitative detection of anti-DENV IgM and anti-DENV IgG antibodies, respectively.

\section{Case definition}

Since we aimed to describe only dengue 4 cases, a dengue (D4) case was defined as a patient confirmed with DENV-4 by RT-PCR. Furthermore, acute DENV-4 cases were classified as primary or secondary infections according to the absence or presence of anti-dengue IgG.

A non dengue (ND) case was defined as a patient with negative results for all dengue biomarkers (viral genome from any serotype, anti-dengue IgM and NS1). 
Patients with negative RT-PCR and Platelia ${ }^{\text {тм }}$ Dengue NS1 Ag-ELISA and/or dengue IgM positive results were considered indeterminate [29]. Indeterminate cases and patients with dengue infection by serotypes other than DENV-4 were excluded.

Laboratory personnel performing one test were blinded to the results of other tests.

\section{Data processing and analysis}

All clinical and laboratory data were collected and recorded in a database using the EpiData ${ }^{\odot} 3.1$ software [30]. Exploratory analysis was performed using the $\operatorname{SPSS}^{\odot}$ v 17.0 software (SPSS Inc., Chicago, Illinois). MedCalc ${ }^{\odot} 14.8 .1$ program was used to calculate $95 \%$ confidence intervals (CI) for sensitivity, specificity, positive predictive value (PPV), negative predictive value (NPV), and likelihood ratios of NS1 Bioeasy ${ }^{\text {TM }}$ and dengue clinical criteria.

A sample size of 137 positive dengue patients was determined as necessary to estimate, with $95 \%$ confidence level, a sensitivity of $85 \%$ with an absolute error of $6 \%$.
Considering a prevalence of $40 \%$ among febrile patients, 342 febrile subjects should be evaluated.

\section{Role of funding source}

The Brazilian National Council of Scientific and Technological Development (CNPq) funded this study (PROEP 402068/2012, REBRATS 401366/2013-8 and Grant level 2 CNPq 311414/2013-3); however, the funder was not involved in the design, sample handling, analysis, data interpretation, conclusions or decision to publish.

\section{Results}

In this study, we evaluated a total of 375 acutely febrile patients. Of those, 38 (10.1\%) were excluded, for reasons shown in Fig. 1. For this reason, 12 patients in the RT-PCR negative group were excluded for presenting positive or indeterminate results for specific dengue IgM or Platelia ${ }^{\mathrm{TM}}$ Dengue NS1 Ag-ELISA (Fig. 1).

From the 325 patients included in the study, 148 (45.5\%) had confirmed DENV-4 infection. No significant differences in age and gender distributions were observed

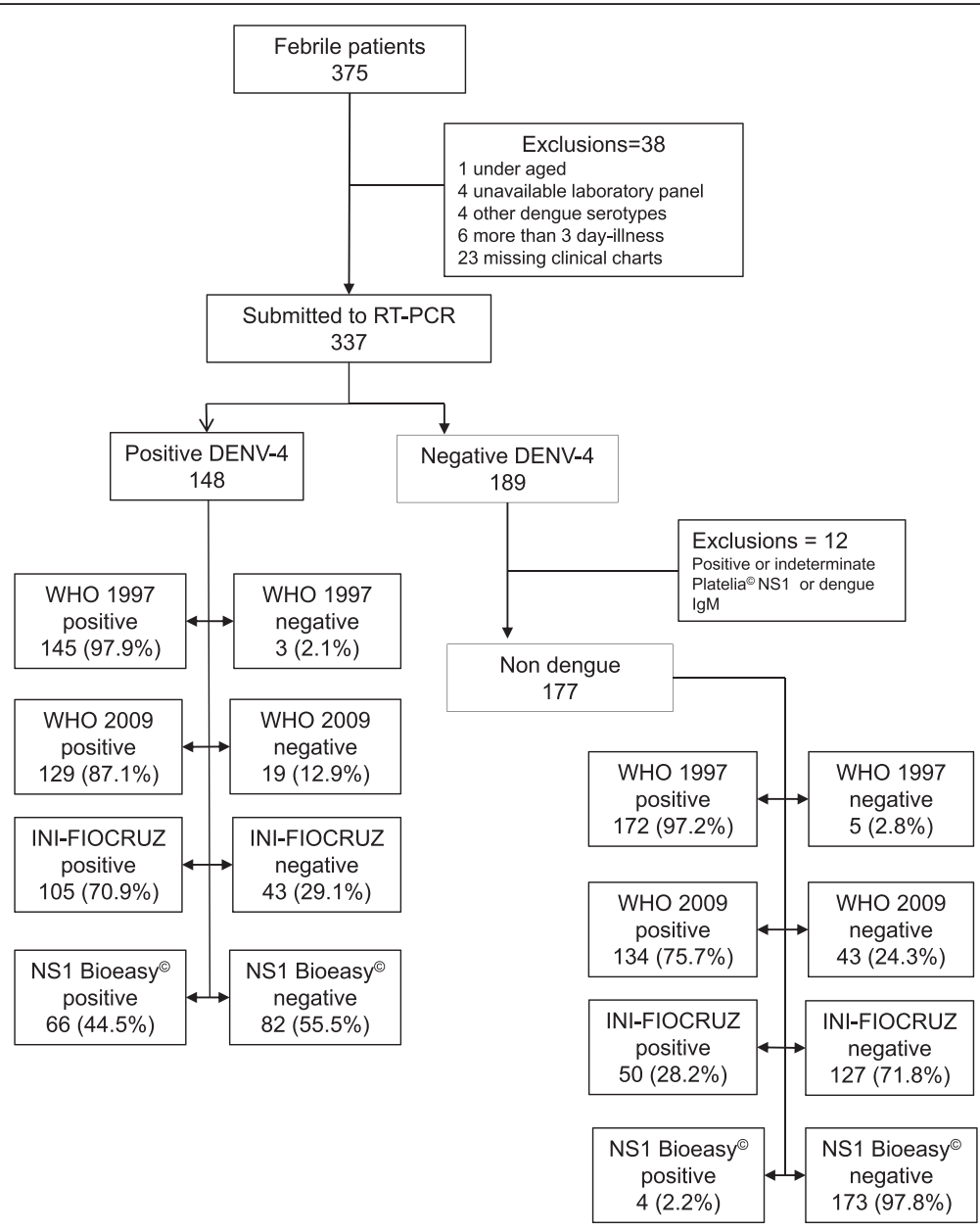

Fig. 1 STARD flow diagram of febrile Rio de Janeiro, March-April, 2013 
between D4 and ND groups. The hematocrits were similar in both groups, but the platelet and leukocyte counts were lower among D4 patients (Table 1). The median time from onset of symptoms and medical care was 2 days in both groups.

The secondary infections, defined by anti-dengue IgG detection in the D4 group, were characterized in 124/ $148(83.7 \%)$ patients, and the NS1 strip test sensitivity was lower $(42 \%)$ in secondary $(52 / 124)$ than in primary dengue cases $(62.5 \%$; 15/24), $p=0.05$.

We also tested the agreement between the NS1 Bioeasy ${ }^{\mathrm{mm}}$ test and the Platelia ${ }^{\text {mw }}$ Dengue NS1 Ag-ELISA. The overall agreement, positive and negative concordances between the tests were $84.1,83.5$ and $84.7 \%$, respectively.

Clinical and laboratory features of eligible patients are shown in Table 2. No sign or symptom alone was able to predict dengue, although leukopenia and thrombocytopenia were more frequent in D4 cases. Leukocyte counts below $4500 / \mathrm{mm}^{3}$ were almost eight times more likely to be present in D4 cases. In our study population, we did not find warning signs such as nuchal rigidity, altered consciousness, and ascites.

Using the WHO 1997 diagnostic criteria, a total of 145 (97.8\%) D4 and 172 (97.2 \%) ND were clinically classified as dengue suspected cases, with a sensitivity of $97.8 \%$ and specificity of $2.8 \%$. The WHO 2009 criteria clinically classified as dengue 129 (87.1 \%) D4 and 134 (75.7 \%) ND with $87.1 \%$ sensitivity and $24.3 \%$ specificity. The INIFIOCRUZ clinical prediction rule classified as dengue suspected 105 (70.9 \%) D4 patients but also 50 (28.2 \%) ND, with a sensitivity of $70.9 \%$ and specificity of $71.8 \%$ (Table 3).

The NS1 Bioeasy ${ }^{\text {min }}$ immunochromatographic strip test alone was more accurate than the clinical criteria (OR = 34.4 ) and showed high specificity (97.8\%). However, the test sensitivity was lower (44.5\%). The test incorrectly classified as ND more than half of dengue patients (55.2 \%). With these results almost a third of the patients with a negative NS1 Bioeasy ${ }^{\mathrm{m}}$ test in our setting were in fact confirmed dengue cases (PPV $=68.3 \%$ ).

Combining the NS1 test with any clinical criteria did not improve the sensitivity or alter the high false negative rates, which remained similar to those obtained by the test alone. But all the evaluated clinical criteria could be complemented by this test resulting in an almost perfect specificity in a clinical setting.

\section{Discussion}

This is the first study we are aware of that assesses the combined accuracy of dengue clinical criteria and a NS1 point-of-care immunoassay for early diagnosis in outpatients during a DENV-4 epidemic. The WHO 1997 criteria was better to rule out the disease. When the INIFIOCRUZ clinical criteria was positive, then the NS1 rapid test should be done. Patients with a positive strip test should be treated as dengue cases; however, negative results should be monitored for dengue or other acute febrile illnesses. As the clinical diagnosis lacks specificity, a definitive dengue case may need laboratory confirmation.

According to Lima, et al. [17], RT-PCR used in the present research showed $90 \%$ sensitivity and more than $95 \%$ specificity on the second day after the onset of disease, the same median time obtained in our sample. It is reasonable to consider this as a good diagnostic tool to confirm dengue fever. However, considering false negative results, we excluded 12 patients with negative RT-PCR and a positive or indeterminate IgM or Platelia ${ }^{\text {Tu }}$ NS1 results in which we could not identify the serotype.

At the time the study population was evaluated, there were no reports of any other Flavivirus circulating simultaneously in the city of Rio de Janeiro that could interfere in dengue laboratory results. We believe that we obtained a true non-dengue set of patients.

We also evaluated the ability of hematological parameters and individual signs and symptoms to discriminate dengue from non-dengue. We found that leukopenia was more frequent in D4 than in ND, although the overlapping range between the two groups prevented an adequate discrimination between them. The relevance of leukopenia as a discriminant feature of dengue infection has been previously documented, although the cutoff values may vary [13, 31-34].

The platelet counts were lower in the D4 group, although true thrombocytopenia was infrequent in both groups. Similar to findings in other studies [22, 35], there was no significant difference in hematocrit between groups. This may be because both hemoconcentration and platelet drop are usually not seen in the first days of disease [34].

Table 1 Epidemiological characteristics of 325 febrile patients, Rio de Janeiro, March-April, 2013

\begin{tabular}{lccc}
\hline & Median (IQR) & & \\
\cline { 2 - 3 } & Dengue 4 (N=148) & Non dengue $(N=177)$ & \\
\hline Age in years & $36.6(23.1-50.1)$ & $46.5(23.0-50.0)$ & 0.99 \\
Hematocrit (\%) & $42.6(40.3-45.0)$ & $42.2(40.0-44.0)$ & 0.45 \\
Leucocyte counts (cells $\left./ \mathrm{mm}^{3}\right)$ & $4251(3400-4667)$ & $7518(5467-7900)$ & $<0.05$ \\
Platelet counts (cells/ $\left.\mathrm{mm}^{3}\right)$ & $217.812(187,667-236,667)$ & $256,4945(221,000-284,667)$ & $<0.05$ \\
\hline
\end{tabular}


Table 2 Clinical and laboratory features of 325 febrile patients, Rio de Janeiro, March-April, 2013

\begin{tabular}{|c|c|c|c|c|c|}
\hline & \multicolumn{2}{|c|}{ Dengue $4(N=148)$} & \multicolumn{2}{|c|}{ Non dengue $(N=177)$} & \multirow[t]{2}{*}{ OR $(95 \% \mathrm{Cl})$} \\
\hline & $N$ & $\%$ & $N$ & $\%$ & \\
\hline \multicolumn{6}{|l|}{ Clinical } \\
\hline Exanthema & 28 & 18.9 & 21 & 11.8 & $1.7(0.9-3.2)$ \\
\hline Conjunctival hyperemia & 75 & 50.6 & 72 & 40.6 & $1.5(1-2.3)$ \\
\hline Lethargy $^{a}$ & 142 & 95.9 & 168 & 94.9 & $1.3(0.4-3.6)$ \\
\hline Joint pain & 110 & 74.3 & 128 & 72.3 & $1.1(0.7-1.8)$ \\
\hline Pain & 143 & 96.6 & 171 & 96.6 & $1.0(0.3-3.4)$ \\
\hline Retro-orbital pain & 105 & 70.9 & 124 & 70.0 & $1.0(0.6-1.7)$ \\
\hline Nausea/vomiting & 99 & 66.9 & 120 & 67.7 & $1.0(0.6-1.5)$ \\
\hline Edema $^{a}$ & 3 & 2.0 & 4 & 2.2 & $0.9(0.2-4.0)$ \\
\hline Liver enlargement $^{\mathrm{a}}$ & 4 & 2.7 & 6 & 3.3 & $0.8(0.2-2.9)$ \\
\hline Persistent vomiting $^{a}$ & 35 & 23.6 & 48 & 27.1 & $0.8(0.5-1.4)$ \\
\hline Myalgia & 134 & 90.5 & 161 & 90.9 & $0.9(0.4-1.9)$ \\
\hline Abdominal tenderness ${ }^{a}$ & 47 & 31.7 & 68 & 38.4 & $0.7(0.5-1.2)$ \\
\hline Bleeding $^{a}$ & 12 & 8.1 & 25 & 14.1 & $0.5(0.3-1.1)$ \\
\hline Headache & 133 & 89.8 & 169 & 95.4 & $0.4(0.2-1.0)$ \\
\hline \multicolumn{6}{|l|}{ Laboratory } \\
\hline Leukopenia & 95 & 64.1 & 33 & 18.6 & $7.8(4.7-13.0)^{b}$ \\
\hline Thrombocytopenia & 17 & 11.4 & 8 & 4.5 & $2.7(1.1-6.5)^{b}$ \\
\hline Anti-dengue lgG & 123 & 83.1 & 161 & 90.9 & $0.5(0.3-1.0)$ \\
\hline
\end{tabular}

$O R$ odds ratio, $\mathrm{Cl}$ confidence interval

${ }^{a}$ warning signs; Leukopenia = leucocyte count $<4,500$ cells $/ \mathrm{mm}^{3}$; thrombocytopenia = platelet count $<150,000$ cells $/ \mathrm{mm}^{3}$

bsignificant odds ratio

Repeated monitoring of the platelet count and hematocrit is recommended, as an abrupt fall in platelet count is proposed as a warning sign, and a significant hematocrit increase is an indirect sign of plasma leakage [10, 34].

Gan, et al. [36] studied 256 adults in Singapore and reported $87.1 \%$ sensitivity and $26 \%$ specificity for the WHO 2009 criteria, similar to the present research. However, the $20 \%$ sensitivity using the WHO 1997 criteria was far different from those obtained in our study.

Due to its lower sensitivity (70.9 \%), the INI-FIOCRUZ clinical criteria does not seem very promising as an exclusive screening alternative. However, it was much more specific than both WHO criteria and showed the best clinical performance for classifying suspected dengue cases. It could be proposed (and tested in a different population) as a screening tool to further confirm using more expensive and time consuming specific laboratory tests.

Among the signs and symptoms in the three sets of clinical criteria, none was significantly more frequent among D4 patients. However, conjunctival hyperemia, a sign used in the INI-FIOCRUZ criteria, had a performance similar to classic signs, such as exanthema. Although not sufficiently accurate to be used as a discriminant feature alone, this was an important finding

Table 3 Accuracy parameters for DENV-4 in 325 febrile patients, Rio de Janeiro, March-April, 2013

\begin{tabular}{llllllll}
\hline & $\begin{array}{l}\text { Sensitivity \% } \\
(95 \% \mathrm{Cl})\end{array}$ & $\begin{array}{l}\text { Specificity \% } \\
(95 \% \mathrm{Cl})\end{array}$ & $\begin{array}{l}\text { LR+ } \\
(95 \% \mathrm{Cl})\end{array}$ & $\begin{array}{l}\text { LR- } \\
(95 \% \mathrm{Cl})\end{array}$ & $\begin{array}{l}\text { PPV } \\
(95 \% \mathrm{Cl})\end{array}$ & $\begin{array}{l}\text { NPV } \\
(95 \% \mathrm{Cl})\end{array}$ & $\begin{array}{l}\text { OR } \\
(95 \% \mathrm{Cl})\end{array}$ \\
\hline WHO 1997 & $97.8(93.8-99.6)$ & $2.8(1.0-6.9)$ & $1.0(1.0-1.1)$ & $0.7(0.2-3.0)$ & $45.6(39.9-51.5)$ & $62.5(24.5-91.5)$ & $1.4(0.3-6.0)$ \\
WHO 2009 & $87.1(80.3-92.1)$ & $24.3(18.1-31.6)$ & $1.1(1.0-1.3)$ & $0.5(0.3-0.9)$ & $48.8(42.4-55.2)$ & $69.5(56.1-80.8)$ & $2.2(1.2-4.0)$ \\
INI-FIOCRUZ & $70.9(63.2-78.6)$ & $71.8(64.5-78.4)$ & $2.5(2.0-3.3)$ & $0.4(0.3-0.5)$ & $67.6(59.5-74.9)$ & $75.3(68.0-81.7)$ & $6.4(3.9-10.4)$ \\
NS1 & $44.5(36.4-53.3)$ & $97.8(94.2-99.4)$ & $19.5(7.3-52.2)$ & $0.6(0.5-0.7)$ & $94.1(85.6-98.4)$ & $68.3(62.1-74.0)$ & $34.4(12.1-97.9)$ \\
WHO 1997 and NS1 & $44.2(35.8-52.9)$ & $97.6(94.0-99.3)$ & $18.3(6.8-49.2)$ & $0.6(0.5-0.7)$ & $93.9(85.0-98.3)$ & $67.8(61.4-73.7)$ & $32.1(11.3-91.4)$ \\
WHO 2009 and NS1 & $40.6(32.3-49.3)$ & $98.2(94.9-99.6)$ & $22.6(7.2-70.6)$ & $0.6(0.5-0.7)$ & $94.9(85.9-98.9)$ & $66.7(60.4-72.5)$ & $37.3(11.3-122.9)$ \\
INI-FIOCRUZ and NS1 & $29.7(22.4-37.8)$ & $98.9(96.0-99.9)$ & $26.2(6.5-106.5)$ & $0.7(0.6-0.8)$ & $95.6(84.9-99.5)$ & $63.2(57.2-68.9)$ & $36.9(8.7-155.5)$ \\
\hline
\end{tabular}

$L R+$ positive likelihood ratio, $L R-$ negative likelihood ratio, $P P V$ positive predictive value, $N P V$ negative predictive value, $O R$ odds ratio, $C l$ confidence interval 
because this clinical sign is not included in the WHO criteria. At least three other studies have reported similar results, both in febrile outpatients in early disease stages [13, 35, 37].

As described by Leo, et al. [38] and Paranavitane, et al. [39] our study targeted early dengue disease in outpatient setting, therefore we could not assess warning signs such as nuchal rigidity, altered consciousness, and ascites, abrupt platelet count fall or hemoconcentration, that occur later during the disease.

The WHO 2009 dengue criteria improved rates of correct identification of severe dengue cases, aiding therapeutics and avoiding unnecessary deaths [30]. However, it did not prove to be much more accurate (sensitive) for screening or clinically diagnosing dengue 4 than the WHO 1997 version. INI-FIOCRUZ criteria showed the best-balanced performance and could enhance specificity being used previously to the NS1 rapid test in selecting a group of patients for this more specific test.

The NS1 Bioeasy ${ }^{\mathrm{mm}}$ showed excellent specificity $(97.8 \%)$ in the study population, which agrees with previous reported results in different countries [15, 40]. However, we found a substantially lower sensitivity $(44.5 \%)$ of Bioeasy ${ }^{\mathrm{mm}}$ NS1 immunoassay in our DENV-4 patients compared to the 61.3 and $90 \%$ sensitivity described respectively by Ferraz, et al. [22], and Silva, et al. [23] in DENV-1 and DENV-3 scenarios. Lower sensitivity to DENV-4 compared to the other serotypes has also been documented by Pal, et al. [15], varying from 42 to $58 \%$ depending on the test used.

Differing performances according to serotype were also recently described in a meta-analysis evaluating two NS1 ELISA tests for early dengue detection [18]. The study concluded that Platelia ${ }^{\mathrm{Tm}}$ NS1 and NS1 Panbio ${ }^{\circ}$ tests showed lower pooled sensitivity for DENV-4 (58 and $37 \%$, respectively) [18]. The interpretation given by some authors includes the NS1 gene polymorphism associated with immunological epitopes, a low NS1 concentration in DENV-4 cases [20, 41], or low overall viremia [21]. Nevertheless, this latter hypothesis was not confirmed by Allonso, et al. [20] in a recent Brazilian study.

As reported in other studies on NS1 detection using conventional ELISA or rapid tests, we observed lower NS1 Bioeasy ${ }^{\mathrm{Tm}}$ sensitivity in secondary $(42 \%)$ compared to primary $(62.5 \%)$ dengue cases $[9,12,15,20]$. As most of the cases $(83.7 \%)$ were secondary infections, this might have contributed to the poor performance of the test in our population. This low performance probably occurred due to a rapid clearance in secondary cases [15] and/or as a result of antigen-antibody complexes that disrupt the test targets impeding the ability of the test to detect free NS1 antigen [42, 43]. Efforts to dissociate immune complexes by acid or heat treatment can probably enhance the assays sensitivities [44].
The high PPV (94.1\%) obtained in this $45.5 \%$ dengue prevalence scenario, similar to that obtained by Pal, et al. [15], indicates that individuals testing positive on NS1 do not require another confirmatory test. The performance on specificity qualifies this test, as a confirmatory one, to streamline the decision to treat the correctly identified dengue patients in the clinical setting. It should be used just after the onset of symptoms in order to avoid complications and deaths and to organize the flow of care at the facility. This rapid test might also be useful for virologic surveillance purposes to select serum samples for RNA detection by PCR.

However, although highly specific, the NS1 rapid test yields high false negative rates (55.5\%) among confirmed DENV-4 patients, and its low sensitivity argues against its incorporation as a screening diagnostic tool in clinical practice. Recently, the Rio de Janeiro State Health Department decided not to include the test for dengue screening in outpatient settings. Its use has been restricted to evaluate critically ill hospitalized patients.

Our study has some limitations: a small sample of primary dengue patients and the absence of the tourniquet test, a WHO sign criteria, considered a painful and lengthy procedure with poor patient compliance. We were also not able to reevaluate the patients in the convalescent period due to the pragmatic diagnostic study outline and the dynamics of the Rio de Janeiro city's health care system. Although the gold standard for dengue diagnosis would be pairing specific dengue IgM in acute and convalescent period, as proposed by WHO [11], recent studies have also exclusively used PCR as the reference test to evaluate early phase dengue [45], to evaluate clinical algorithms [46] or ELISA and NS1 tests [15].

\section{Conclusions}

The development of more sensitive point-of-care tests for dengue diagnosis to act as a screening tool remains a challenge, especially for endemic countries, where the prevalence of secondary cases is high. In summary, we proposed a clinical-laboratory algorithm which combines the INI-FIOCRUZ and the WHO 1997 clinical criteria, in order to select ambulatory suspected early dengue cases (within 3 days of onset of disease) eligible for NS1 strip testing. The patients that test positive should be immediately treated for dengue, and the patients that test negative would still require subsequent diagnostic investigation. We recommend external validation in different settings.

\section{Abbreviations}

Cl: confidence interval; CNPq: Brazilian National Council of Scientific and Technological Development; D4: dengue 4; DENV-1: dengue virus serotype 1; DENV-2: dengue virus serotype 2; DENV-3: dengue virus serotype 3; DENV4: dengue virus serotype 4; DNA: deoxyribonucleic acid; ELISA: enzyme-linked immunosorbent assay; FIOCRUZ: Oswaldo Cruz Foundation; 
IgG: immunoglobulin G; IgM: immunoglobulin M; INI-FIOCRUZ: Evandro Chagas National Institute of Infectious Diseases, Oswaldo Cruz Foundation; IQR: interquartile range; LR+: positive likelihood ratio; LR-: negative likelihood ratio; ND: non-dengue; NPV: negative predictive value; NS1: nonstructural protein-1; OR: odds ratio; PPV: positive predictive value; RNA: ribonucleic acid; RT-PCR: reverse transcriptase-polymerase chain reaction.; STARD: Standards for Reporting of Diagnostic Accuracy Study; UPA 24H: Unidade de Pronto Atendimento; WHO: World Health Organization.

\section{Competing interests}

The authors declare that they have no competing interests.

\section{Authors' contributions}

SNB, SRLP and RPD conceived of the study, participated in its design and coordination and helped to draft the manuscript. DNRO and FMQ participated in its design, data collection and recording. YHMH participated in the study design, data analysis and interpretation and helped to draft the manuscript. RMRN and FBS carried out the immunoassays, rapid immunochromatographic test, viral characterization and writing the manuscript. CNC participated in the design of the study and performed the statistical analysis. All authors read and approved the final manuscript.

\section{Acknowledgements}

We thank Dr Maria Angélica Borges dos Santos for her helpful discussions reviewing this manuscript, Dr Monique da Rocha Queiroz Lima for executing all the specific laboratory assays, Allan and Kate Crean for the careful English editing.

\section{Author details}

${ }^{1}$ Laboratory of Clinical Epidemiology, Evandro Chagas National Institute of Infectious Diseases, Oswaldo Cruz Foundation, Rio de Janeiro, Brazil. ${ }^{2}$ National School of Public Health, Oswaldo Cruz Foundation, Rio de Janeiro, Brazil. ${ }^{3}$ Germano Sinval Faria Teaching Primary Care Center, National School of Public Health, Oswaldo Cruz Foundation, Rio de Janeiro, Brazil. ${ }^{4}$ Flavivirus Laboratory, Oswaldo Cruz Institute, Oswaldo Cruz Foundation, Rio de Janeiro, Brazil.

Received: 19 August 2015 Accepted: 22 January 2016

Published online: 29 January 2016

\section{References}

1. World Health Organization. Dengue: diagnosis, treatment, prevention and control. Geneva: WHO; 2009. http://www.who.int/neglected_diseases/en/. Accessed in 15 Jan 2015

2. Bhatt $\mathrm{S}$, Gething PW, Brady OJ, Messina JP, Farlow AW, Moyes $\mathrm{CL}$, et al. The global distribution and burden of dengue. Nature. 2013;496(7446):504-7.

3. San Martin JL, Brathwaite O, Zambrano B, Solorzano JO, Bouckenooghe A, Dayan $\mathrm{GH}$, et al. The epidemiology of dengue in the Americas over the last three decades: a worrisome reality. Am J Trop Med Hyg. 2010;82(1):128-35.

4. Dietz VJ, Gubler DJ, Rigau-Perez JG, Pinheiro F, Schatzmayr HG, Bailey R, et al. Epidemic dengue 1 in Brazil, 1986: evaluation of a clinically based dengue surveillance system. Am J Epidemiol. 1990;131(4):693-701.

5. Nogueira RM, Miagostovich MP, Lampe E, Schatzmayr HG. Isolation of dengue virus type 2 in Rio de Janeiro. Mem Inst Oswaldo Cruz. 1990;85(2):253.

6. Nogueira RM, Schatzmayr HG, de Filippis AM, dos Santos FB, da Cunha RV, Coelho JO, et al. Dengue virus type 3, Brazil, 2002. Emerg Infect Dis. 2005; 11(9):1376-81.

7. Teixeira MG, Costa Mda C, Barreto F, Barreto ML. Dengue: twenty-five years since reemergence in Brazil. Cad Saude Publica. 2009;25 Suppl 1:S7-18.

8. Nogueira RM, Eppinghaus AL. Dengue virus type 4 arrives in the state of Rio de Janeiro: a challenge for epidemiological surveillance and control. Mem Inst Oswaldo Cruz. 2011;106(3):255-6.

9. Hang VT, Nguyet NM, Trung DT, Tricou V, Yoksan S, Dung NM, et al. Diagnostic accuracy of NS1 ELISA and lateral flow rapid tests for dengue sensitivity, specificity and relationship to viraemia and antibody responses. PLoS Negl Trop Dis. 2009;3(1), e360.

10. Brasil. Ministério da Saúde. Secretaria de Vigilância em Saúde. Diretoria Técnica de Gestão. Dengue : diagnóstico e manejo clínico - adulto e criança / Ministério da Saúde, Secretaria de Vigilância em Saúde, Diretoria Técnica de Gestão. - 4. ed. - Brasília : Ministério da Saúde, 2011.
11. World Health Organization. Dengue haemorrhagic fever: diagnosis, treatment, prevention and control. Genebra: WHO; 1997. http://www.who. int/neglected_diseases/en/. Accessed in 15 Nov 2014.

12. Chaterii S, Allen Jr JC, Chow A, Leo YS, Ooi EE. Evaluation of the NS1 rapid test and the WHO dengue classification schemes for use as bedside diagnosis of acute dengue fever in adults. Am J Trop Med Hyg. 2011;84(2):224-8.

13. Daumas RP, Passos SR, Oliveira RV, Nogueira RM, Georg I, Marzochi KB, et al. Clinical and laboratory features that discriminate dengue from other febrile illnesses: a diagnostic accuracy study in Rio de Janeiro, Brazil. BMC Infect Dis. 2013;13:77.

14. Lima MRQ, Nogueira RM, Santos FB. Dengue diagnosis: commercially available kits and laboratory support. In: Clinical insights: dengue: transmission, diagnosis \& surveillance. 1st ed. London: Future Medicine Ltd; 2014. p. 27-47.

15. Pal S, Dauner AL, Mitra I, Forshey BM, Garcia P, Morrison AC, et al. Evaluation of dengue NS1 antigen rapid tests and ELISA kits using clinical samples. PLoS One. 2014;9(11), e113411.

16. Domingo C, Alves MJ, de Ory F, Teichmann A, Schmitz $H$, Muller R, et al. International external quality control assessment for the serological diagnosis of dengue infections. BMC Infect Dis. 2015;15:167.

17. Lima MR, Nogueira RM, Schatzmayr HG, dos Santos FB. Comparison of three commercially available dengue NS1 antigen capture assays for acute diagnosis of dengue in Brazil. PLoS Negl Trop Dis. 2010;4(7), e738.

18. da Costa VG, Marques-Silva AC, Moreli ML. A meta-analysis of the diagnostic accuracy of two commercial NS1 antigen ELISA tests for early dengue virus detection. PLoS One. 2014;9(4), e94655.

19. Fry SR, Meyer M, Semple MG, Simmons CP, Sekaran SD, Huang JX, et al. The diagnostic sensitivity of dengue rapid test assays is significantly enhanced by using a combined antigen and antibody testing approach. PLoS Negl Trop Dis. 2011;5(6), e1199.

20. Allonso D, Meneses MD, Fernandes CA, Ferreira DF, Mohana-Borges R. Assessing positivity and circulating levels of NS1 in samples from a 2012 dengue outbreak in Rio de Janeiro, Brazil. PLoS One. 2014;9(11), e113634.

21. Sea VR, Cruz AC, Gurgel RQ, Nunes BT, Silva EV, Dolabella SS, et al. Underreporting of Dengue-4 in Brazil due to low sensitivity of the NS1 Ag test in routine control programs. PLoS One. 2013;8(5), e64056.

22. Ferraz FO, Bomfim MR, Totola AH, Avila TV, Cisalpino D, Pessanha JE, et al. Evaluation of laboratory tests for dengue diagnosis in clinical specimens from consecutive patients with suspected dengue in Belo Horizonte, Brazil. J Clin Virol. 2013;58(1):41-6.

23. Silva FG, Silva SJS, Rocco IM, Silveira VR, Suzuki A, Katz G, et al. Evaluation of commercial kits for detecting the antigen NS1-dengue- São Paulo. Bepa. 2011;8(9):14-26.

24. Bossuyt PM, Reitsma JB, Bruns DE, Gatsonis CA, Glasziou PP, Irwig LM, et al. Toward complete and accurate reporting of studies of diagnostic accuracy. The STARD initiative. Am J Clin Pathol. 2003;119(1):18-22

25. Daumas RP, Brasil P, Bressan CS, Oliveira RV, Carvalho BB, Carneiro DV, et al. Interobserver agreement on signs and symptoms of patients with acute febrile illness. Infection. 2011;39(2):135-40.

26. Gutierrez G, Gresh L, Perez MA, Elizondo D, Aviles W, Kuan G, et al. Evaluation of the diagnostic utility of the traditional and revised $\mathrm{WHO}$ dengue case definitions. PLoS Negl Trop Dis. 2013;7(8), e2385.

27. Failace R. Hemograma: manual de interpretação. 5th ed. Artmed: Porto Alegre; 2009.

28. Lanciotti RS, Calisher CH, Gubler DJ, Chang GJ, Vorndam AV. Rapid detection and typing of dengue viruses from clinical samples by using reverse transcriptase-polymerase chain reaction. J Clin Microbiol. 1992;30(3):545-51.

29. Setiati TE, Mairuhu AT, Koraka P, Supriatna M, Mac Gillavry MR, Brandjes DP, et al. Dengue disease severity in Indonesian children: an evaluation of the World Health Organization classification system. BMC Infect Dis. 2007;7:22.

30. Lauritsen JM, Bruus M. EpiData 3ed. A comprehensive tool for validated entry and documentation of data. Odense, Denmark: The EpiData Association; 2003.

31. Gregory CJ, Lorenzi OD, Colon L, Garcia AS, Santiago LM, Rivera RC, et al. Utility of the tourniquet test and the white blood cell count to differentiate dengue among acute febrile illnesses in the emergency room. PLoS Negl Trop Dis. 2011;5(12), e1400.

32. Macedo GA, Gonin ML, Pone SM, Cruz OG, Nobre FF, Brasil P. Sensitivity and specificity of the World Health Organization dengue classification schemes for severe dengue assessment in children in Rio de Janeiro. PLoS One. 2014;9(4), e96314. 
33. Horstick O, Jaenisch T, Martinez E, Kroeger A, See LL, Farrar J, et al. Comparing the usefulness of the 1997 and 2009 WHO dengue case classification: a systematic literature review. Am J Trop Med Hyg. 2014:91(3):621-34.

34. Huang SY, Lee IK, Wang L, Liu JW, Hung SC, Chen CC, et al. Use of simple clinical and laboratory predictors to differentiate influenza from dengue and other febrile illnesses in the emergency room. BMC Infect Dis. 2014;14:623.

35. Low JG, Ooi EE, Tolfvenstam T, Leo YS, Hibberd ML, Ng LC, et al. Early Dengue infection and outcome study (EDEN) - study design and preliminary findings. Ann Acad Med Singapore. 2006;35(11):783-9.

36. Gan VC, Lye DC, Thein TL, Dimatatac F, Tan AS, Leo YS. Implications of discordance in world health organization 1997 and 2009 dengue classifications in adult dengue. PLoS One. 2013;8(4), e60946.

37. Conroy AL, Gelvez M, Hawkes M, Rajwans N, Liles WC, Villar-Centeno LA, et al. Host biomarkers distinguish dengue from leptospirosis in Colombia: a case-control study. BMC Infect Dis. 2014;14:35.

38. Leo YS, Gan VC, Ng EL, Hao Y, Ng LC, Pok KY, et al. Utility of warning signs in guiding admission and predicting severe disease in adult dengue. BMC Infect Dis. 2013;13:498.

39. Paranavitane SA, Gomes L, Kamaladasa A, Adikari TN, Wickramasinghe N, Jeewandara C, et al. Dengue NS1 antigen as a marker of severe clinical disease. BMC Infect Dis. 2014;14:570.

40. Tan LK, Lye DC, Pok KY, Mok SQ, Chua RC, et al. Diagnosing dengue at the point-of-care: utility of a rapid combined diagnostic kit in Singapore. PLoS One. 2014;9(3), e90037.

41. Colombo TE, Vedovello D, Araki CS, Cogo-Moreira H, dos Santos IN, Reis AF, et al. Dengue-4 false negative results by Panbio ${ }^{\circ}$ Dengue Early ELISA assay in Brazil. J Clin Virol. 2013;58(4):710-2.

42. Peeling RW, Artsob H, Pelegrino JL, Buchy P, Cardosa MJ, Devi S, et al. Evaluation of diagnostic tests: dengue. Nat Rev Microbiol. 2010;8(12 Suppl):S30-8.

43. Blacksell SD. Commercial dengue rapid diagnostic tests for point-of-care application: recent evaluations and future needs? J Biomed Biotechnol. 2012;2012:151967.

44. Lima MR, Nogueira RM, Filippis AM, Nunes PC, Sousa CS, Silva MH, et al. A simple heat dissociation method increases significantly the ELISA detection sensitivity of the nonstructural-1 glycoprotein in patients infected with DENV type-4. J Virol Methods. 2014;204:105-8.

45. Nujum ZT, Thomas A, Vijayakumar K, Nair RR, Pillai MR, Indu PS, et al. Comparative performance of the probable case definitions of dengue by WHO (2009) and the WHO-SEAR expert group (2011). Pathog Glob Health. 2014;108(2):103-10.

46. Cecilia D, Kakade M, Alagarasu K, Patil J, Salunke A, Parashar D, et al. Development of a multiplex real-time RT-PCR assay for simultaneous detection of dengue and chikungunya viruses. Arch Virol. 2015;160(1):323-7.

\section{Submit your next manuscript to BioMed Central and we will help you at every step:}

- We accept pre-submission inquiries

- Our selector tool helps you to find the most relevant journal

- We provide round the clock customer support

- Convenient online submission

- Thorough peer review

- Inclusion in PubMed and all major indexing services

- Maximum visibility for your research

Submit your manuscript at www.biomedcentral.com/submit

C Biomed Central 